\title{
Obstacle Avoidance Technology Based on Collision Cone for UAV Fast SLAM
}

\author{
Zeng-xiang Yang ${ }^{1 \mathrm{a}}$, Xi-bin Wang ${ }^{2 \mathrm{~b}}$, Wei-chao $\mathrm{Si}^{3 \mathrm{c}}$ \\ ${ }^{1}$ Wenling Power Supply Company of Bay Power Supply State Wenling Power Supply Company, \\ Taizhou Zhejing, 317500, China \\ ${ }^{2}$ Department of Control Engineering, Naval Aeronautical and Astronautical University, Yantai \\ Shandong 264001, China \\ ${ }^{3}$ Department of Armament Science and Technology, Naval Aeronautical and Astronautical \\ University, Yantai Shandong 264001, China \\ aYangzengxiang520@163.com, 'bangxi_bin@163.com, 9luchdevilsi@163.com
}

\begin{abstract}
Keywords: collision cone; simultaneous location and mapping; uninhabited aerial vehicle; obstacle avoidance

Abstract: To solve the obstacle avoidance problem when UAV is implementing SLAM, a collision cone is built using obstacle avoidance strategy based on collision cone. By ascertaining whether the velocity direction of UAV motion is within the cone, the occasion of UAV obstacle avoidance is judged. This algorithm is simulated and tested based on two dimension built motion model of UAV. The result shows that this algorithm not only complishes SLAM, estimating the position of UAV and obstacles, but also achieving better obstacle avoidance effects.
\end{abstract}

\section{Introduction}

When UAV is carrying out the mission of simultaneous location and mapping (SLAM) ${ }^{[1]}$, there are many solid or moving obstacles in the unknown environment, so the obstacle avoidance is the problem which must be considered in UAV SLAM. Now in the most research of SLAM, the position of obstacles are estimated ${ }^{[2,3]}$, but the obstacle avoidance is not considered. In reference ${ }^{[4-6]}$ the obstacle avoidance is researched, but the pose of UAV or the position of obstacles is known beforehand. Therefore, the research of obstacle avoidance on UAV SLAM is very few, and becomes the imperative task of SLAM.

Kumar and Ghose ${ }^{[7]}$ proposed one navigation and guidance rule to accomplish the mission of route point tracking and obstacle avoidance simultaneously. However this algorithm used the distance information of radar and flight zones restricted in the two dimension plane. Radar sensors were used in Kwag Kang ${ }^{[8]}$ paper.

Binocular vision sensors are applied on the UAV in this paper, and the states of every obstacle are estimated from the two dimension vision information. Since the vision measurements are nonlinear function of relative state, the UAV obstacle avoidance technology is researched based on collision cone and simulated in the framework of EKFSLAM. The result shows that this algorithm can succeed in avoiding the threat of obstacles, at the same time carrying out the mission of SLAM, therefore, ensuring the flight safety for UAV.

\section{Artificial potential field method}

The basic ideas of artificial potential field are that the motion of UAV in the environment is viewed as a virtual motion of artificial field. Obstacles exclude UAV, becoming the exclusion field which is as low as UAV is far from obstacles. Goal attracts UAV, becoming attraction field. Attraction field and exclusion field act on UAV together, making UAV tone velocity and direction continuously, gradually approach and arrive at the goal. 
Shown as figure 1 , position of UAV arriving is $p_{G}$, and obstacles in the environment are $O_{i}(i=1,2, \mathrm{~L}, n)$. Artificial field $U_{a r t}(p)$ of UAV at position $p$ can be denoted as the sum of goal field $U_{G}(p)$ and obstacle field $U_{o}(p)$, therefore, the abstract force acting on UAV $F(p)$ is:

$$
F_{G}(p)+\sum_{i=1}^{n} F_{o_{i}}(p)=-\nabla U_{G}(p)+\sum_{i=1}^{n}\left(-\nabla U_{O_{i}}(p)\right)
$$

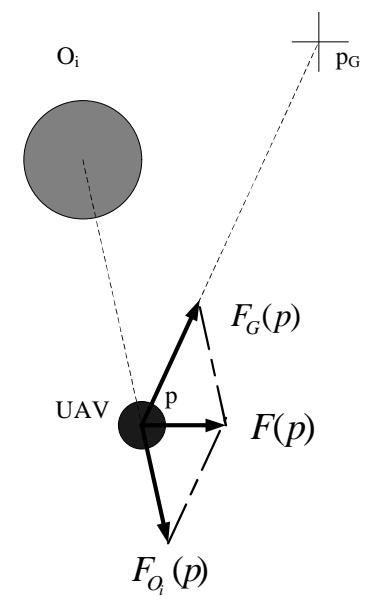

Fig. 1 Sketch of UAV force in artificial potential field

Selection of potential field function is agile. Goal potential and obstacle potential field functions that $\mathrm{O}$. Khatib build are as follows:

$$
U_{G}(p)=\frac{1}{2} k\left(p-p_{G}\right)^{2}, U_{o}(p)= \begin{cases}\frac{1}{2} \eta\left(\frac{1}{\rho}-\frac{1}{\rho_{0}}\right)^{2}, & \rho \leq \rho_{0} \\ 0 & , \rho>\rho_{0}\end{cases}
$$

In which, $k 、 \eta>0, \rho$ is the shortest distance between UAV and obstacles, and $\rho_{0}$ is the threshold.

The structure of potential field is simple and easy for real time obstacle avoidance, but it also has limit. For example, when the resultant force of all obstacles and goal is zero, UAV may not move, trapping in local extreme. If goal is near to obstacle, and UAV approaches the goal and obstacle simultaneously, when the exclusion force is larger than the attraction, UAV may not arrive at the goal. To conquer these problems, many improved potential field methods are proposed.

\section{Obstacle Avoidance Technology Based on Collision Cone}

Once estimating the position of the obstacles in the SLAM for UAV, one obstacle avoidance standard is needed to detect whether the obstacles causes threat.

Chakravarthy and Ghose ${ }^{[9]}$ proposed one standard built by two dimension collision cone and collision cone is determined for every obstacle if the relative velocity vectors are located in the collision cone, the obstacle is viewed dangerous. Collision cone is expanded three dimension space in the reference ${ }^{[10]}$. UAV is required to keep a minimal distance $d$ in the obstacle avoidance problem. Therefore, collision/safe borderline is a sphere that radius is $d$ and the circle center is located in the centre of obstacle, then collision cone is determined by the vertical line set from UAV to collision cone $^{[11]}$.

At time $\mathrm{k}, \mathrm{UAV}$ position vector is $X_{v_{k}}$ and velocity vector is $V_{v_{k}}$. For an obstacle located at $X_{o b s}$, $X_{k}=X_{o b s}-X_{v_{k}}$ is the relative position. If considering the two dimension plane built by relative position vector $X_{k}$ and relative velocity vector $V_{v_{k}}$, the collision cone is precisely determined by two vector $\left(p_{1}, p_{2}\right)$ from the UAV position and vertical to borderline circle in figure 1. $p_{1}$ and $p_{2}$ are represented in the following: $p_{i}=X_{k}+d u_{i}, \quad i=1,2$ 
$u_{1}$ and $u_{2}$ are the unit vector from the obstacle position to terminals $p_{1}$ and $p_{2}$ separately:

$\left\{\begin{array}{l}\left.u_{1}=-\frac{1}{\left\|X_{k}\right\|^{2}}\left(c\left(X_{k} \cdot V_{v_{k}}\right)+d\right) X_{k}+c V_{v_{k}}\right) \\ \left.u_{2}=\frac{1}{\left\|X_{k}\right\|^{2}}\left(c\left(X_{k} \cdot V_{v_{k}}\right)-d\right) X_{k}-c V_{v_{k}}\right)\end{array}\right.$

In which, $c=\sqrt{\frac{\left\|X_{k}\right\|^{2}-d^{2}}{\left\|X_{k}\right\|^{2}\left\|V_{v_{k}}\right\|^{2}-\left(X_{k} \cdot V_{v_{k}}\right)^{2}}}$

UAV velocity is written the vectors concerning $p_{1}$ and $p_{2}: V_{v_{k}}=a p_{1}+b p_{2}$

The coefficients $a$ and $b$ are calculated in the following.

$a=\frac{1}{2}\left(\frac{X_{k} \cdot V_{v_{k}}}{\left\|X_{k}\right\|^{2}-d^{2}}+\frac{1}{c d}\right), b=\frac{1}{2}\left(\frac{X_{k} \cdot V_{v_{k}}}{\left\|X_{k}\right\|^{2}-d^{2}}-\frac{1}{c d}\right)$

Then collision cone is given according to the following formula:

$$
a>0 \text { and } b>0
$$

When above formula is satisfied, the vehicle is regarded in the danger of collision and avoidance is adopted by determining the goal $X_{a p}$ in the figure 1 . The goal $X_{a p}$ position is in the following:

$X_{a p}=\left[\begin{array}{l}X_{a p} \\ Y_{a p} \\ Z_{a p}\end{array}\right]= \begin{cases}p_{1}, & 0<b \leq a \\ p_{2}, & 0<a<b\end{cases}$

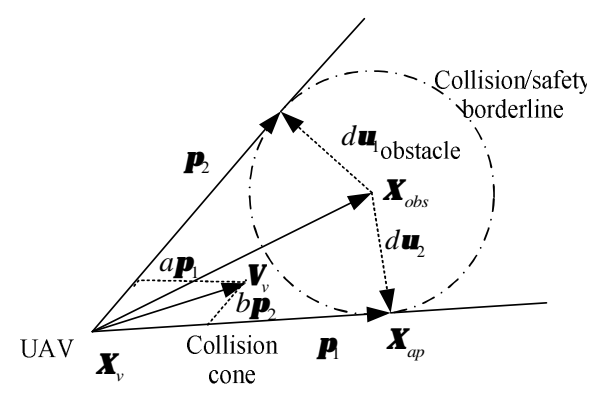

Fig. 1 Collision cone and aiming point

Since vehicle has the constant velocity aiming to the goal, time arriving at the goal is derived in the following: $t_{g o}=t_{k}+\frac{X_{a p}-X_{v_{k}}}{U_{v_{k}}}$

When $t_{g o}-t_{k}$ is larger than the given threshold $T$, obstacle avoidance is not urgent for UAV. Also, if $t_{g o}-t_{k}$ is negative and $t_{g o}$ is larger than $t_{f}$, then collision is no chance. So, in addition to collision cone, the following arrival time is augmented:

$$
t_{g o}-t_{k}<T \text { and } 0<t_{g o}<t_{f}
$$

If formula (1) and (2) are satisfied, the obstacle is regarded urgent. If there are several obstacles, the one with shortest arrival time is selected urgent obstacles.

After determining the urgent avoidance obstacle, UAV must be adopted to mobile or stop immediately, but emergency braking is always late, making that UAV must move $\gamma$ angle to escape the obstacles: $X_{k} \cdot V_{v_{k}}=\left|X_{k}\right|\left|V_{v_{k}}\right| \cos \gamma$

Since the main value range of reverse cosine is $0 \sim \pi$ and UAV moved deflexion angle $\gamma$ of collision avoidance for UAV is often not larger than $\pi / 2$, computed deflexion angle $\gamma$ is : 
$\gamma=\arccos \left(\frac{X_{k} \cdot V_{v_{k}}}{\left|X_{k}\right|\left|V_{v_{k}}\right|}\right)$

If $\gamma>\pi / 2$, then $\gamma=\gamma-\pi$

That is $\begin{cases}\gamma=\gamma, & 0 \leq \gamma<\pi / 2 \\ \gamma=\gamma-\pi, & \pi / 2 \leq \gamma<\pi\end{cases}$

Considering the effect of control and sensors measurement noise simultaneously, UAV deflexion angle $\gamma$ should be a little lager than above computed $\gamma$, that is to add a safe factor $\eta$ for $\gamma$ :

$\gamma^{*}=\eta \gamma$

In which, $\eta$ is a constant larger than 1 .

\section{UAV Plane Motion Model}

Simulated model uses the simplified UAV plane two-dimension model, shown by figure 2.

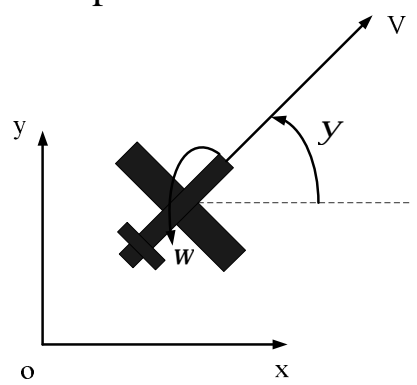

Fig. 2 UAV two dimensions model

UAV states $X_{v}=\left[\begin{array}{lll}x & y & \psi\end{array}\right]^{T}, x$ and $y$ are position of UAV locating on two dimension plane, $\psi$ is azimuth angle, control $U=\left[\begin{array}{ll}v & \gamma\end{array}\right]^{T}, v$ is the speed of UAV motion, $\gamma$ is the change of azimuth. The change of state variables is as follows:

$\left\{\begin{array}{c}x_{k}=x_{k-1}+v d t \cos \left(\psi_{k-1}+\gamma\right) \\ y_{k}=y_{k-1}+v d t \sin \left(\psi_{k-1}+\gamma\right) \\ \psi_{k}=\psi_{k-1}+\gamma\end{array}\right.$

UAV state equation is: $X_{v_{k}}=f\left(X_{v_{k-1}}, U_{k}\right)+W_{k}$

The state of the $i$ th landmark is $m_{i}=\left[\begin{array}{ll}m_{x} & m_{y}\end{array}\right]^{T}$, assuming the landmarks are all still: $m_{i, k}=m_{i, k-1}$.

The measurement equation of UAV observes $i$ th landmark is as follows: $Z_{i, k}=h\left(X_{v_{k}}, m_{i, k}\right)+w_{k}$

\section{Simulation test}

In the test, UAV uses plane two-dimension model and the control variables are velocity $v$ and change of azimuth $\gamma$ in the figure 3. At the initial time, UAV is moving at the velocity of $5 \mathrm{~m} / \mathrm{s}$ along the direction of the goal $(180,0)$ in the position $(20,0)$, and the view field of camera is $90^{\circ}$. To simplify the complexity, there exist two obstacles in the position of $(60,5)$ and $(120,0)$ with the size of $2 \mathrm{~m}$ radius circle. The minimal distance is $10 \mathrm{~m}$ from UAV to the goal, and system noise covariance $Q$ and measurement covariance $R$ are separately:

$$
Q=\operatorname{diag}\left\{0.3^{2},(0.02 \pi / 180)^{2}\right\}, R=\operatorname{diag}\left\{0.1^{2},(0.01 \pi / 180)^{2}\right\}
$$

Threshold $T$ is $4 \mathrm{~s}$ and safe factor $\eta$ selects 1.25 . Figure 4 shows the UAV obstacle avoidance curve based on collision cone. Magenta hexagon denotes UAV motion goal; green star points denote obstacle centre; blue real line denotes UAV true obstacle avoidance curves and red dashed line denotes estimated UAV obstacle avoidance curve. 


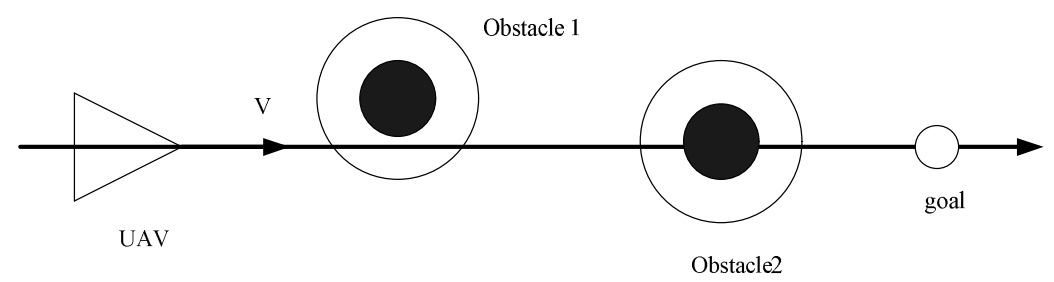

Fig. 3 UAV initial position and obstacles distribution

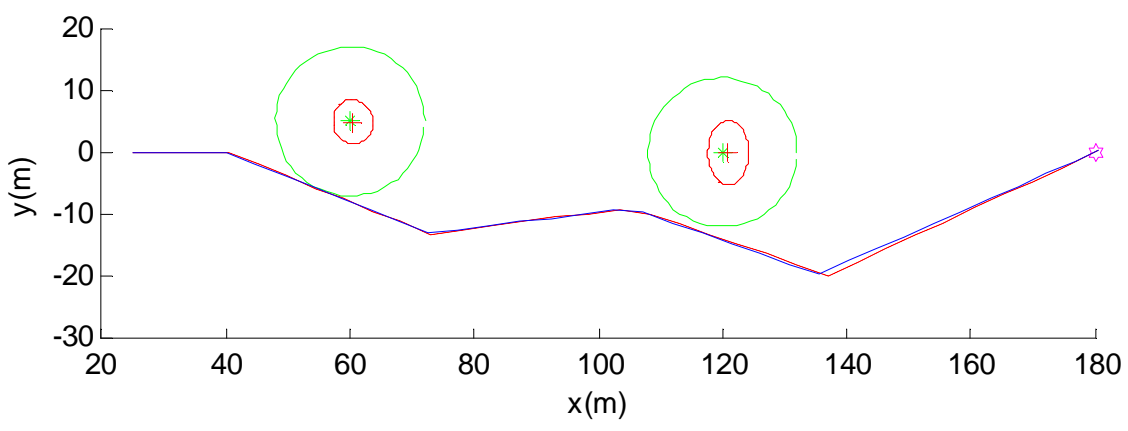

Fig. 4 UAV SLAM obstacle avoidance route planning based on collision cone

From the simulation of figure 4, the error is little between the UAV estimated position and planed trajectory with no noise. The obstacle centre positions are well estimated and UAV escapes the two obstacles successfully to arrive at the goal with the error less than $2 \mathrm{~m}$.

\section{Conclusions}

This paper researches the trajectory planning problem of on the conditions of UAV SLAM obstacle avoidance. Since the SLAM environment for UAV is unknown, there are still or moving obstacles inevitably when UAV is completing the missions, and real time planning the UAV trajectory is needed to escape the obstacles in the environment. Obstacle avoidance strategy is used based on collision cone, and UAV obstacle avoidance motion is by deciding whether UAV velocity direction is in the collision cone. For the built two dimension model, simulation is carried out for the strategy, and The result shows that this algorithm can succeed in avoiding the threat of obstacles, at the same time carrying out the mission of SLAM.

\section{References}

[1] R Smith, M Self, P Cheeseman. Estimating uncertain relationships in robotics[C]. Proceedings of Conference on Uncertainty in Artificial Intelligence. Amsterdam: 1988, 435-461.

[2] Gregory F. Ivey, Eric N. Johnson. Investigation of methods for simultaneous localization and mapping using vision sensors[C]. AIAA Guidance, Navigation and Control Conference and Exhibit, Colorado: AIAA, 2006

[3] S Sukkarich, H Durrant-Whyte. Towards the development of simultaneous localisation and map building for an unmanned air vehicle[C]. Proc. Int. Conference of Field and Service Robotics, 2001: 193-200,

[4] Zhao Jing, Chen Yi-min. Mobile robot route programming and obstacles avoidance based on binocular vision [J]. Computer engineering and designing. 2009, 30 (23): 5462-5466

[5] Wang Guang-jun, Tian Jin-wen, Liu Jian. Application of Laser image radar in the process of aircraft avoiding obstacles [J]. Radio science journal. 2001, 16(2):249-251

[6] Wang Yi-fan, Kan De-rong, Zhang Li-yang. A fast parallax distance measurement method for micro UAV obstacle avoidance [J]. Chinese test technology, 2008, 34(3):114-116 
[7] B. A. Kumar and D. Ghose. Radar-Assisted Collision Avoidance/Guidance Strategy for Planar Flight.[C]. IEEE Transactions on Aerospace and Electronic Systems. Vol.37, No.1. January 2001.

[8] Y. K. Kwag and J. W. Kang. Obstacle Awareness and Collision Avoidance Radar Sensor System for Low-Altitude FlyingSmart UAV[C]. Digital Avionics Systems Conference. October 2004.

[9] A. Chakravarthy and D. Ghose. Obstacle Avoidance in a Dynamic Environment: A Collision Cone Approach[J]. IEEE Transactions on Systems, Man and Cybernetics-Part A: Systems and Humans. Vol. 28, No.5. 1998.

[10] Y. Watanabe, A. J. Calise, E. N. Johnson and J. H. Evers. Minimum-Effort Guidance for Vision-Based Collision Avoidance[C]. AIAA Atmospheric Flight Mechanics Conference. August 2006.

[11] Yoko Watanabe, Anthony J. Calisey and Eric N. Johnso. Vision-Based Obstacle Avoidance for UAVs[C]. AIAA Guidance, Navigation and Control Conference and Exhibit. Hilton Head, South Carolina, 2007 